\title{
The Sunspot Activity in the Last Two Millenia on the Basis of Indirect and Instrumental Indexes: Time Series Models and Their Extrapolations for the 21st Century
}

\author{
Boris Komitov $^{1}$ and Vladimir Kaftan ${ }^{2}$ \\ ${ }^{1}$ Institute of Astronomy, Bulgarian Academy of Sciences, PO Box 39, Bulgaria, \\ 6003 Stara Zagora; komitov@mbox.digsys.bg \\ ${ }^{2}$ Central Research Institute of Geodesy, Aerial Surveying and Cartography, Federal Agency of \\ Geodesy and Cartography, Moscow, Russia. kaftan@geod.ru
}

In the present study a time series analysis of three of the most well-known Sun activity data series is made: 1) the Hoyt-Schatten (Group Sunspot Number $R g$ )(Hoyt \& Schatten 1988); 2) the Schove and 3) the Greenland (Dye-3 ice probe) 'cosmogenic' ${ }^{10} B e$ concentration series (Schove 1983, Beer et al. 1998). The series "1" is based on instrumental observations for the last $\sim 400$ years. The series "2" is a reconstruction of all SchwabeWolf cycle magnitudes after AD 296 by use of historical reports mainly for auroras and naked-eye visible sunspots. The series " 3 " is an indicator for the processes in outer solar corona and interplanetary space for the epoch AD 1423-1985. Two independent methods for time series analysis are used: 1 ) the $T$ - $R$ periodogramm analysis (Komitov, 1986); 2 ) the method of Kaftan (2002).

In all three series the cycles of 80-90, 100, 110-130, $\sim 200$ and 360-400 years as well as the quasy-11-year cycle are present. Unlike in the Schove and Hoyt-Schatten series, in the Greenland beryllium data a powerful 65-67-year cycle is well observed. There are statistically significant quasy- 20-22, 26-27 and 50-55-year cycles too. This is not typical of the instrumental sunspot data series like the Hoyt-Schatten $(R g)$ and Zürich $(R i)$ series, but it is very close to some climatic and geomagnetic indexes' behaviour. The large-scale time variation structures of the three series are in good accordance with each other.

However, there are significant differences between the instrumental sunspot and beryllium data for the Maunder minimum (1640-1720 AD). On the other hand, evidence that there are no significant differences between Schove's and the $R g$ series during the same epoch is given. These results pointed out that the "cosmogenic" isothopes series are not the perfect indicator for the active processes in the Sun's photosphere and particulary - for the sunspot activity. In some "extremal" epochs (such as the Maunder minimum) the relationship between the solar wind and photospheric activity processes can be not very close and it can be statistically insignificant.

Qualitative differences between Maunder's and Dalton's supercenturial solar minimums is established on the basis of ${ }^{10} \mathrm{Be}$. During the Maunder minimum a significant 22-year oscilation exists, but the 11-year cycle is totally absent. For the epoch 1790-1830 AD (the Dalton minimum) the 22-year oscilation is absent in beryllium data, but a very weak signal of 11-year cycle is present. This is an indirect evidence against the hypotesis for the "lost" 11-year cycle at the beginning of the Dalton minimum. 


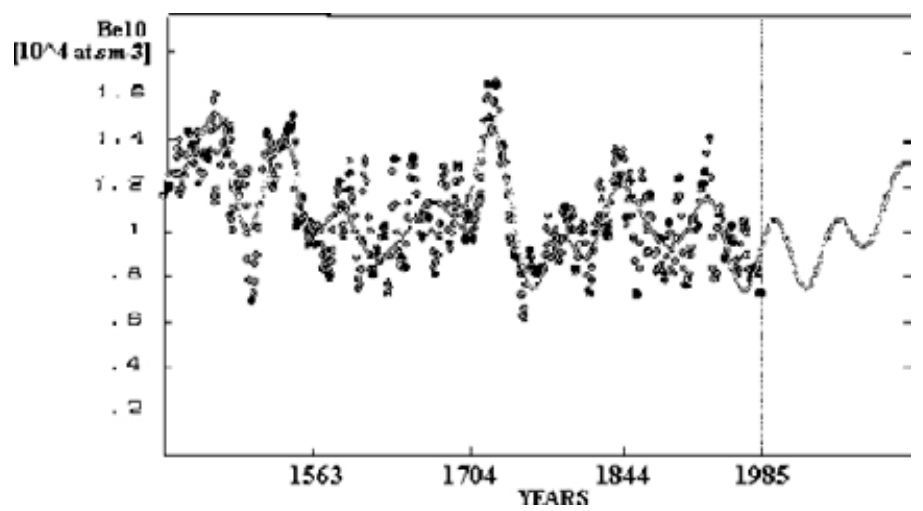

Figure 1. A model of the "greenland" ${ }^{10} \mathrm{Be}$ time series (the dotted curve) for the epoch (AD 1423-1985). Cycles of duration of 52, 65, 108 and 192 years and a non-linear trend are included. The model is extrapolated after AD 1985. The general tendency of incresing ${ }^{10} \mathrm{Be}$ concentrations during the 21st century corresponds to the decrease in solar activity (supercenturial solar minimum).

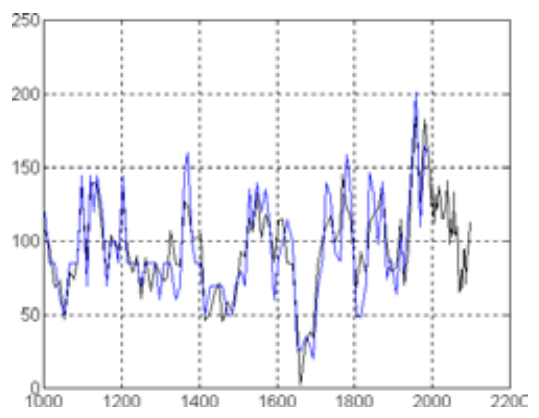

Figure 2. The Schove series (blue line) for the last millenia and its model, obtained by the method of V. Kaftan (black line). The model is extrapolated after AD 1996. The forecoming supercenturial solar minimum during the 21 st century is clearly shown (see also Komitov \& Kaftan 2003).

In the opposite of the epochs of supercenturial minimums, during the supercenturial solar maximum (1944-1985) both 11- and 22-year cycles are very strong in the ${ }^{10} \mathrm{Be}$ series.

Models for all three investigated series are built on the basis of the time series analysis. It follows from their extrapolations for the 21st century that a supercenturial solar minimum will be occuring during the next few decades (fig. 1 and 2). It will be similar in magnitude to the Dalton minimum, but probably longer as the last one.

\section{References}

Beer, J., Tobias, S. and Weiss, N.: 1998, An Active Sun throughout the Maunder Minimum, Solar Phys. 181(1), 237-249.

Hoyt, D. and Schatten, K.: 1998, Group Sunspot Numbers: A New Solar Activity Reconstruction, Solar Phys. 181(2), 491-512.

Kaftan, V. I.: 2002, Gravity Variation at the Moscow Fiducial Station, IAG pp. 293-296.

Komitov, B.: 1986, On a Possible Effect of Solar Cycles on the Climate of Bulgaria, number 5 in Solar Data, pp. 73-78.

Komitov, B. P. and Kaftan, V.: 2003, Solar Activity Variations for the Last Millenia. Will the Next Long-Period Solar Minimum be Formed?, Geom. \& A Aeron. 43, 553.

Schove, D. J.: 1983, Sunspot Cycles, Stroudsburg: Hutchinson Ross. 